\title{
Proyección de la demanda de materiales de construcción en Colombia por medio de análisis de flujos de materiales y dinámica de sistemas
}

\author{
Juan Pablo Ríos Ocampo* \\ Yris Olaya Morales ${ }^{* * *}$ \\ Gabriel Jaime Rivera León ${ }^{* * * *}$
}

\author{
Recibido: 04/08/2015 - Aceptado: 29/11/2016 \\ DOI: 10.22395/rium.v16n31a4
}

\begin{abstract}
Resumen
En este artículo se estima la demanda futura de materiales de construcción en Colombia y de los minerales necesarios para producirlos usando dinámica de sistemas y análisis de flujos de materiales. Por medio del análisis de flujos de materiales se determinan las cantidades de minerales requeridos para la producción de cemento y concreto. La producción requerida de cemento y concreto se estima por medio de un modelo de dinámica de sistemas el cual se ajusta con datos del censo de construcción del DANE. Los resultados indican que, sin tener en cuenta la oferta secundaria de materiales, entre 2014 y 2017 se requerirían 176.9 millones de toneladas de agregados triturados, arena de río, cemento gris, roca muerta y cerámica cocida. Para la fabricación del cemento y concreto, se necesitarían 15.4 millones de toneladas de caliza, 14,9 de grava, 14,1 de yeso, 13,3, 9,7 y 9,3 de arcilla, minerales de hierro y arena, respectivamente

Palabras clave: análisis del flujo de materiales; cemento; demanda de materiales de construcción; dinámica de sistemas; hormigón; proyección.
\end{abstract}

\footnotetext{
* Artículo generado en el proyecto de investigación Análisis de flujos dinámicos de materiales aplicado a la proyección de demanda de materiales de construcción en Colombia, desarrollado con recursos de la Convocatoria 617 de 2013 Jóvenes Investigadores de Colciencias. Fecha de realización del proyecto: abril de 2014 a abril de 2015. Entidades que financian: Departamento Administrativo de Ciencia, Tecnología e Innovación (Colciencias), Colombia, y la Facultad de Minas de la Universidad Nacional de Colombia sede Medellín.

** Economista, estudiante de Maestría en Ingeniería de Sistemas de la Universidad Nacional de Colombia sede Medellín. Joven Investigador de Colciencias.jprioso@unal.edu.co

*** M. Sc. en Ingeniería de Sistemas, Ph. D. Mineral Economics, Colorado School of Mines. Profesora asociada, Departamento de Ciencias de la Computación y la Decisión, Facultad de Minas, Universidad Nacional de Colombia sede Medellín. yolayam@unal.edu.co

**** Ingeniero Industrial, estudiante de Maestría en Ingeniería Administrativa de la Universidad Nacional de Colombia sede Medellín. Joven Investigador de Colciencias, Colombia. gjriveral@unal.edu.co
} 


\title{
Projection of the demand of construction materials in Colombia through the analysis of materials flows and systems dynamic
}

\begin{abstract}
This article provides an estimation of the future demand of construction materials in Colombia and necessary minerals to produce them by using systems dynamic and analysis of materials flows. Through the analysis of materials flows the amount of minerals required for the production of cement and concrete are determined. Required production of cement and concrete is estimated through a model of systems dynamic, which is adjusted with data from DANE construction census. Results indicate that, disregarding the secondary offer of materials, 176.9 million tons of ground aggregates, river sand, gray cement, dead rock, and cooked ceramic would be required. Manufacture of cement and concrete would require 15.4 million tons of lime, 14.9 million tons of gravel, 14.1 million tons of gypsum, and 13.3, 9.7, and 9.3 million tons of clay, iron minerals, and sand, respectively.
\end{abstract}

Keywords: materials flow analysis; systems dynamic; demand of construction materials; projection; cement; concrete.

\section{Projeção da demanda de materiais de construção na Colômbia por meio da análise de fluxos de materiais e dinâmica de sistemas}

\begin{abstract}
Resumo
Neste artigo, estima-se a demanda futura de materiais de construção na Colômbia e dos minerais necessários para produzi-los usando dinâmica de sistemas e análise de fluxos de materiais. Por meio da análise de fluxos de materiais, determinam-se as quantidades de minerais requeridos para a produção de cimento e concreto. A produção exigida de cimento e concreto estima-se por meio de um modelo de dinâmica de sistemas que se ajusta aos dados do censo de construção do Departamento Administrativo Nacional de Estatística (Dane). Os resultados indicam que, sem considerar a oferta secundária de materiais, entre 2014 e 2017, foram requeridos 176,9 milhões de toneladas de agregados triturados, areia de rio, cimento cinzento, rocha morta e cerâmica cozida. Para a fabricação do cimento e do concreto, seriam necessários 15,4 milhões de toneladas de calcário, 14,9 de cascalho, 14,1 de gesso, 13,3, 9,7 e 9,3 de argila, minerais de ferro e areia.
\end{abstract}

Palavras-chave: análise do fluxo de materiais; cimento; demanda de materiais de construção; dinâmica de sistemas; concreto armado; projeção. 


\section{INTRODUCCIÓN}

Además de ser uno de los sectores más representativos y dinámicos de la economía colombiana, el sector de la construcción es un importante consumidor de materiales y generador de residuos [1, 2]. Entre 2000 y 2011 el consumo de materiales para la fabricación de cemento y productos de hormigón, cemento y yeso aumentó en 190\% y $310 \%$, respectivamente [3]. Se espera que las tasas de crecimiento se mantengan en los próximos 10 años para apoyar la construcción de nuevas viviendas y obras de infraestructura [4] y que la demanda de cemento supere los 12 millones de toneladas en 2022 [5]. Dadas las expectativas de crecimiento de la construcción en Colombia, la estimación de la demanda de materiales es necesaria para la planeación adecuada tanto en el sector minero como en la cadena de suministro de materiales y la disposición de residuos.

Puesto que hay restricciones técnicas, ambientales, sociales y legales en la cadena de suministro de materiales de construcción, es importante identificar los factores que determinan tanto la demanda de materiales como sus cambios, y aplicar metodologías de proyección adecuadas al comportamiento de la demanda.

La UPME [6] estima la demanda y oferta futura de materiales de construcción en Colombia basándose en la proyección de la demanda de edificaciones residenciales y no residenciales y obras civiles. En esta metodología, la demanda futura de materiales se encuentra aplicando factores de consumo a la demanda de edificaciones, esta última expresada en metros cuadrados construidos. Para proyectar la demanda de edificaciones, se ajusta un modelo VAR multivariado donde los metros cuadrados construidos dependen de variables socioeconómicas como población, hogares, tasa de formación de hogares, PIB per cápita, desempleo, créditos aprobados, licencias de construcción y censo de obras. Este modelo auto-regresivo multivariado captura la dinámica de la construcción, y es usado para generar proyecciones a 10 años.

A pesar de su utilidad para realizar proyecciones de corto y mediano plazo, la metodología usada por la UPME [6] no considera aspectos importantes de la oferta y demanda de materiales de construcción como la oferta secundaria de materiales, representada en el reciclaje, la cual depende del inventario acumulado de materiales.

En este artículo se presenta un análisis del consumo, utilización y demanda futura de materiales dentro del sector de la construcción en Colombia en el cual se incorpora la oferta secundaria de materiales y los materiales ya acumulados en la infraestructura construida. El análisis presentado se basa en la metodología de Análisis del Flujo de Materiales (AFM), inicialmente propuesta por Ayres [7], y estandarizada como método de análisis por Eurostat [8, 9], y en Dinámica de Sistemas [10]. 
El análisis de flujos de materiales (AFM) consiste en el estudio de los flujos físicos de recursos naturales y materiales en un sistema determinado. El término AFM se extiende a una familia de enfoques analíticos y herramientas de medida que se basan en la contabilidad de los flujos en unidades físicas y el principio de balance físico de materia [11]. El AFM es aplicado para realizar desde análisis económicos de flujos de materiales para estudiar los flujos de un grupo de materiales determinado en una economía hasta métodos de análisis de ciclo de vida para analizar los flujos de materiales en un producto específico [12]. En Colombia el AFM ha sido aplicado de manera agregada para analizar flujos históricos de combustibles fósiles, biomasa, minerales industriales y materiales de construcción [13]; sin embargo, no se encuentra evidencia de aplicaciones para el sector de la construcción.

La dinámica de sistemas, inicialmente por Forrester en 1961, identifica los flujos de materiales e información que se acumulan dentro de los sistemas y los cuales pueden estar sujetos a retardos. El comportamiento dinámico del sistema resulta de la interacción entre las acumulaciones (niveles) y los flujos variables, la cual se representa por medio de ecuaciones diferenciales $[10,14]$.

\section{FLUJOS DE MATERIALES DE CONSTRUCCIÓN EN COLOMBIA EN 2013}

Para este análisis se establecen los límites del sistema desde la entrada de las materias primas (ya extraídas) necesarias para la preparación de cemento y concreto hasta la salida de cemento y concreto hacia los sectores de la construcción. Estos límites se fijan porque el cemento es representativo tanto para la demanda de materias primas como para la oferta de materiales de construcción en Colombia. Dado que este estudio se enfoca en el suministro de minerales, se excluyen del análisis los flujos de agua, aire y energía.

En el diagrama Sankey de la figura 1 se muestran los flujos de materiales de construcción usados en 2013 para la elaboración de clínker, cemento y concreto en Colombia. La información sobre el nivel de producción y de despachos (por destino) del cemento y la preparación de concreto y sus usos se obtuvo del DANE [15] y CAMACOL [16]. Por su parte, la producción de caliza, minerales de hierro y clinker se obtuvo de SIMCO [17]. Los flujos de los demás materiales se estimaron con base en los estándares fijados por Eurostat [9] y los informes técnicos de EPA [18], la UPME [6] y WBCSD [19], ver tabla 1. 
Tabla 1: Flujos del ciclo del clínker, cemento y concreto.

\begin{tabular}{|c|c|c|c|c|}
\hline Flujo & Información & Unidades & Reporte & Fuente \\
\hline Clínker & Producción de clínker & Toneladas & Mensual & {$[17]$} \\
\hline Caliza & $80 \%$ de clínker & Toneladas & Mensual & {$[17]$} \\
\hline Arcilla & $10 \%$ de clínker & Toneladas & & {$[18,27]$} \\
\hline Minerales de hierro & 7,94\% de clínker & Toneladas & Anual & {$[17]$} \\
\hline Otros materiales - clínker & $2,06 \%$ & Toneladas & & {$[18]$} \\
\hline Residuos & $\begin{array}{l}\text { Por 1,8 toneladas de materias primas } \\
\text { se produce } 1 \text { tonelada de clinker; } 1.5 \\
\text { corresponde a caliza }\end{array}$ & Toneladas & & {$[20]$} \\
\hline Cemento & Producción de cemento & Toneladas & Mensual & {$[15,16]$} \\
\hline Despacho cemento & total cemento consumido y por destino & Toneladas & Mensual & {$[15,16]$} \\
\hline Inventario de cemento & $\begin{array}{l}\text { Diferencia - producción y despacho de } \\
\text { cemento }\end{array}$ & Toneladas & Mensual & {$[15]$} \\
\hline Yeso & $5 \%$ y $11 \%$ - producción de cemento & Toneladas & & [19] \\
\hline Otros materiales - cemento & $9,5 \%$ - producción de cemento & Toneladas & & {$[21]$} \\
\hline clinker & $79,5 \%$ - producción de cemento* & Toneladas & Mensual & {$[17,20]$} \\
\hline Usos & Despachos por destino & Toneladas & Mensual & {$[15]$} \\
\hline Concreto & $\begin{array}{l}\text { Producción de concreto** -concreteras, } \\
\text { fibrocemento, prefabricadas }\end{array}$ & \begin{tabular}{|l|}
$\mathrm{m}^{3}$, \\
Kilogramos \\
\end{tabular} & Trimestral & {$[15]$} \\
\hline Grava & $41 \%$ - producción de concreto & Toneladas & & {$[21]$} \\
\hline Cemento & $11 \%$ - producción de concreto & Toneladas & & {$[21]$} \\
\hline Arena & $26 \%$ - producción de concreto & Toneladas & & {$[21]$} \\
\hline Agua & $16 \%$ - producción de concreto & Toneladas & & {$[21]$} \\
\hline Aire & $6 \%$ - producción de concreto & Toneladas & & {$[21]$} \\
\hline VIS & *** & Toneladas & Mensual & {$[15]$} \\
\hline No VIS & $* * *$ & Toneladas & Mensual & {$[15]$} \\
\hline Obras civiles & "*** & Toneladas & Mensual & {$[15]$} \\
\hline Edificaciones & *** & Toneladas & Mensual & {$[15]$} \\
\hline Otras - uso cemento & *** & Toneladas & Mensual & {$[15]$} \\
\hline
\end{tabular}

* Al comparar la producción de Clínker y de Cemento reportado por el SIMCO, se obtiene un relación promedio de 79,5\%. Teniendo en cuenta los datos reportados por el trabajo EPA [21] se tienen en cuenta más materiales y el Yeso tiene una mayor participación.

** Con base en el informe de CEMEX, Concreto de resistencia a 28 días convencional, la producción de cemento reportado en $\mathrm{m}^{3}$ se halló la densidad total teniendo en cuenta $2.550 \mathrm{~kg} / \mathrm{m}^{3}$ Concreto de resistencia a 28 días convencional. 
Como se observa en la figura 1, los principales materiales usados para fabricar clinker son caliza, minerales de hierro y arcilla. Por cada tonelada de clinker, se consumen en total unas 1,8 toneladas de materias primas [20]. En 2013, en Colombia la fabricación de clinker consumió 16.09 millones de toneladas de materiales entre arcilla, hierro y caliza. Del total de materiales consumidos, el 55\% (8,94 millones de toneladas) se transformó en clinker y el restante 45\% (7,16 millones de toneladas) se emitió en forma de $\mathrm{CO}_{2}$.

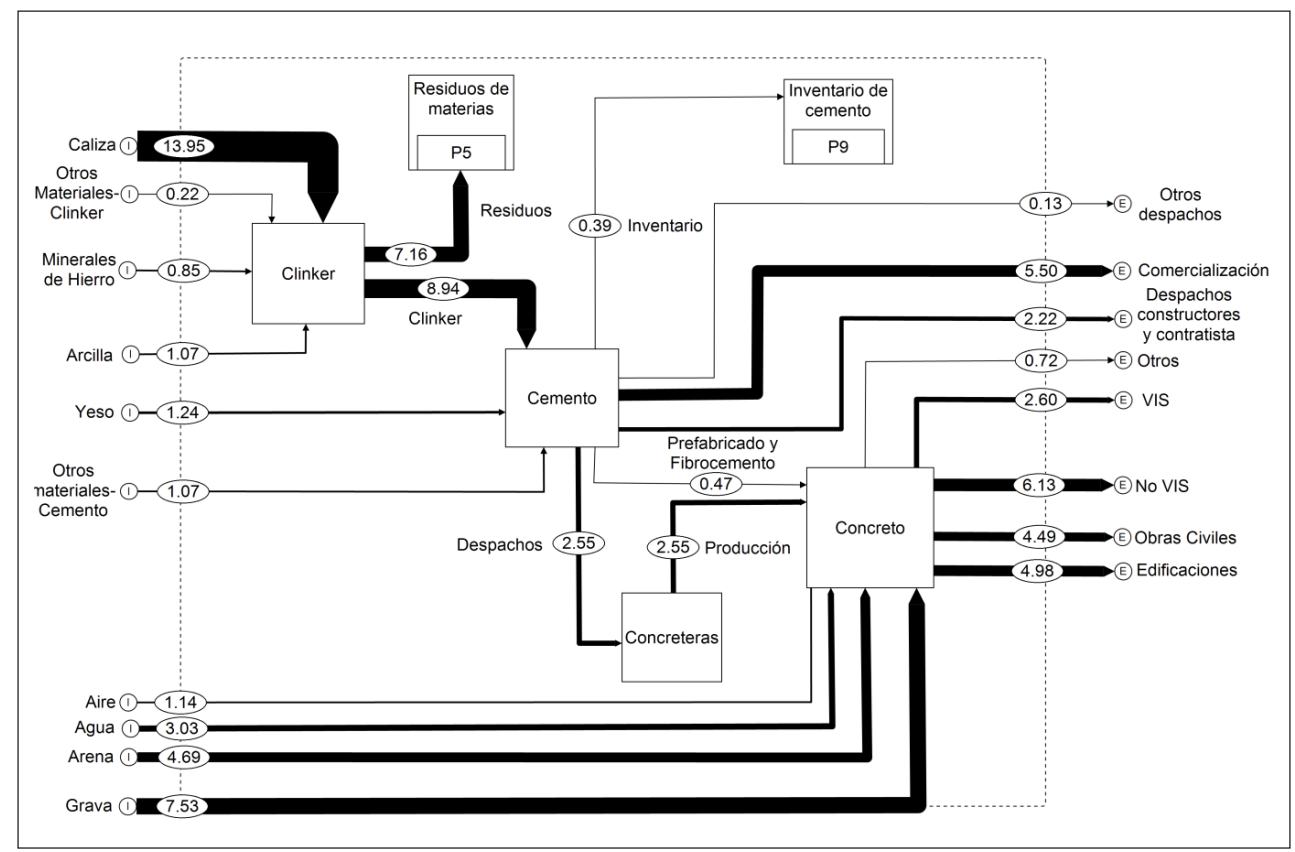

Figura 1: Proceso productivo del cemento y el concreto para el 2013.

Fuente: [15]- [19], [21]- [22]. Elaboración propia. Cifras en millones de toneladas.

El clinker producido en Colombia se usa en su totalidad en la preparación de cemento. En 2013 la fabricación de cemento consumió 11.25 millones de toneladas de materiales entre clinker, yeso y otros materiales; esta cantidad corresponde a los despachos de cemento reportados por el DANE durante el 2013 [15].

En conjunto, el 53\% de las materias primas usadas en 2013 se destinó a la fabricación de clinker y cemento, y el $47 \%$ restante a la fabricación de concreto. De los flujos de salida del cemento se despacharon 10.87 millones de toneladas a las concreteras, comercializadores, constructores, y contratistas y a la fabricación de prefabricados y fibrocemento. E1 28\% del cemento despachado (3,02 millones de tonelada) se destinó a la fabricación de concreto en concreteras, prefabricados y fibrocemento. 
Además del flujo de entrada de cemento, la producción de concreto requiere 4.69 millones de toneladas de arena y 7.53 millones de toneladas de grava, que representan un total de 19,41 millones de toneladas de materias primas, incluyendo el aire y el agua. El consumo de agua y aire solo se consideró para la preparación del concreto. En la fabricación de clinker y cemento se usan agua y aire para refrigeración, pero estos materiales no entran en la composición de los productos por lo que no se incluyeron en el análisis.

Se desconoce la producción de concreto de los demás destinatarios del cemento, por lo que los 18.92 millones de toneladas de concreto producido se toman como flujos finales del sistema. El flujo correspondiente a la diferencia entre el cemento producido y el despachado genera un inventario de 0.39 millones de toneladas.

En cuanto al destino final de concreto, un $34 \%$ va a viviendas diferentes a las de interés social (No VIS), $25 \%$ para obras civiles y $27 \%$ para edificaciones. De otra parte, por cada tonelada de concreto despachada se demandaron 0,72 toneladas de caliza, $0,24,0,38$ y 0,44 toneladas de arena, grava y otros materiales, respectivamente. De estas demandas, el 18\% tuvo como destino final la construcción de No VIS; el 14\%, a edificaciones; el 13\%, a las obras civiles, y el $8 \%$, VIS.

De acuerdo con las estimaciones realizadas, en Colombia se demandaron 34.783.480 toneladas de materiales en el 2013. La estimación hecha es superior en un $10 \%$ a los 31.383 .000 toneladas que la UPME [6] estima como la demanda de materiales para el mismo año, en las principales ocho ciudades del país. La diferencia entre nuestra estimación y el dato publicado por la UPME se debe a que en este estudio se tomó en cuenta el total de cemento producido en el país y el censo de edificaciones para 12 áreas urbanas, tres metropolitanas (datos reportados por el DANE [22, 23]). Por su parte, el estudio de la UPME se centra en analizar los materiales de construcción, principalmente la arcilla, en las principales ocho ciudades del país.

Debido a que los datos del DANE no discriminan los flujos de cemento gris por sector de destino (viviendas, edificaciones y obras civiles), en la figura 1 se dividen por canal de distribución. No obstante, tal como se muestra en la figura, la construcción de viviendas es un destino importante para el cemento y el concreto, y recibe cerca del $47 \%$ del concreto producido. Por la importancia de la construcción de vivienda en la demanda de materiales, en la siguiente sección enfocamos el análisis en la dinámica de la construcción de edificaciones.

\section{DINÁMICA DE LA ACTIVIDAD CONSTRUCTORA EN COLOMBIA}

La actividad constructora está estrechamente relacionada con el ciclo económico [24] y también exhibe una dinámica propia como resultado de los retardos de construcción 
y de información en los mercados inmobiliarios. Como se vio en la figura 1 el 52\% de los materiales de construcción en Colombia se destina a la vivienda, y por tal razón, los modelos presentados a continuación se enfocan en la acumulación de materiales como resultado de la construcción de vivienda.

Se propone un modelo de Dinámica de Sistemas para la actividad constructora y la acumulación de materiales. La metodología de dinámica de sistemas sigue un proceso iterativo para definir el problema de estudio. En la fase de definición, se representa el problema a través de diagramas causales y diagramas de flujos y niveles. Los diagramas causales identifican las variables endógenas y exógenas que componen el sistema y cuya interacción produce el comportamiento dinámico de este.

Luego de definir el problema, las relaciones de causalidad entre las variables se expresan matemáticamente como ecuaciones diferenciales no lineales, las cuales reflejan la complejidad y los ciclos asociados al sistema. Las ecuaciones diferenciales expresan de forma matemática los procesos de flujo y acumulación que también se representan por medio de diagramas de flujos y niveles.

Las ecuaciones diferenciales que componen el modelo incluyen los retardos que hacen parte del sistema. Una vez completado el modelo, el sistema de ecuaciones se resuelve numéricamente. De esta forma, se puede entender la consecuencia de las decisiones a lo largo del tiempo [10].

\subsection{Modelo de dinámica de la construcción y el consumo de materiales}

Las figuras 2 y 3 muestran los diagramas de flujos y niveles del modelo de construcción y acumulación de materiales. Las variables de los diagramas se basan en las definiciones del Censo de Edificaciones del DANE [2, 23]. Las variables de nivel (rectángulos) entre las que están las obras en proceso, finalizadas, paralizadas son acumulaciones que aumentan o disminuyen de acuerdo con los flujos que entran o salen de ellas. Los flujos de entrada o salida afectan los niveles periódicamente. Es decir, las obras nuevas que se inician cada trimestre incrementan el nivel de obras en proceso. Igualmente, las obras culminadas son un flujo de salida que reduce el nivel de obras en construcción cada semestre. Las variables auxiliares y los parámetros necesarios para definir los flujos se representan como círculos y rombos, respectivamente.

Las obras nuevas alimentan las obras en construcción. Las obras inician a una tasa que depende de variables económicas exógenas las cuales son proyectadas con métodos estadísticos (sección 2.2). Las obras iniciadas alimentan la variable nivel obras en construcción. Las obras en construcción pasan a ser obras finalizadas a una tasa que depende del tiempo de construcción; sin embargo, un porcentaje de las obras 
en construcción se suspende y reinicia por distintas razones cada período; esto se representa por medio de la variable de nivel Paralizadas (figura 2).

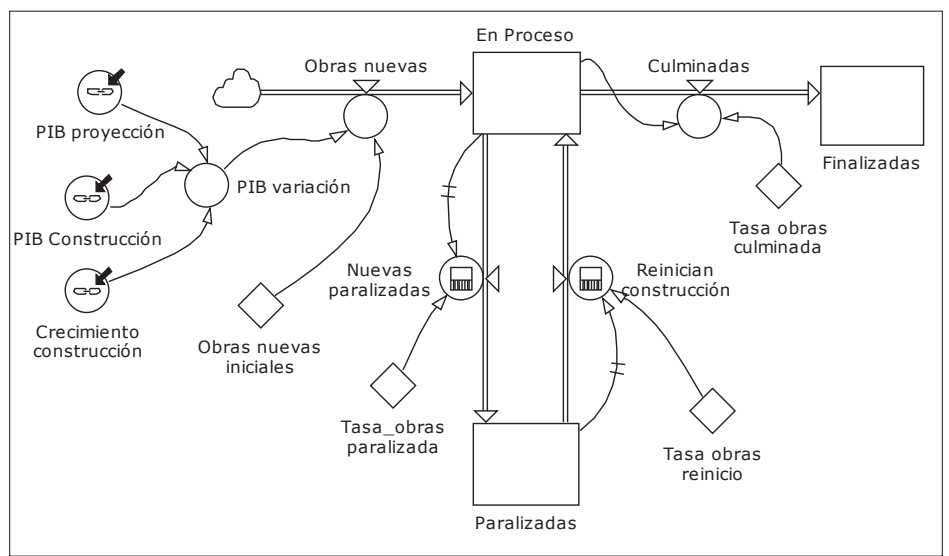

Figura 2: Diagrama de flujos y niveles del proceso de construcción.

Fuente: DANE [3, 24], elaboración propia

La figura 3 representa acumulación de materiales en el sector de la construcción y sus principales flujos. La variable de nivel materiales aumenta cada periodo por la actividad constructora y disminuye con las demoliciones. Las demoliciones liberan residuos; un porcentaje de estos se recicla y la reutilización de materiales disminuye la demanda de nuevos insumos. En este modelo, el reciclaje está definido por los materiales liberados por cada sistema constructivo y una tasa de reciclaje. Las ecuaciones del sistema son:

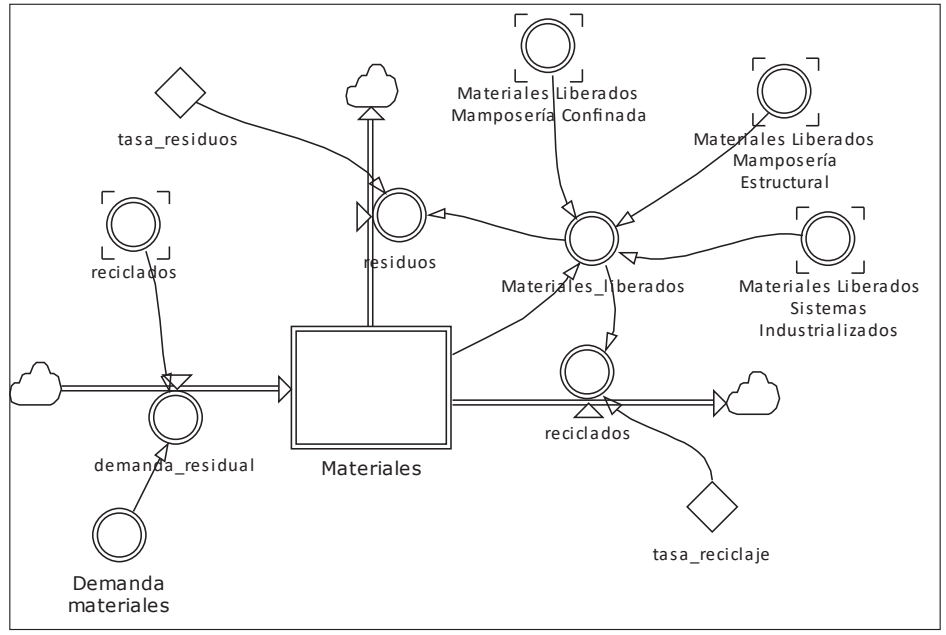

Figura 3: Diagrama de flujos y niveles de materiales de construcción.

Fuente: DANE [3, 23], elaboración propia 
La ecuación 1 representa la variable de nivel en construcción que corresponde a las construcciones activas en un período específico. Este nivel, expresado en metros cuadrados, aumenta con la entrada de obras nuevas $(\mathrm{ON})$ y de obras que reinician actividades (RC) y disminuye con las obras que son culminadas (C) y las nuevas obras paralizadas (NP).

$$
\text { En_construcción }=\int(O N+R C)-(C+N P) d t
$$

La variable de nivel Paralizadas, ecuación 2, refleja la cantidad de obras (en metros cuadrados) que no presentan actividad productiva durante un período de tiempo. Este nivel aumenta con las nuevas obras paralizadas (NP) y disminuye con las obras que reinician construcción $(\mathrm{RC})$.

$$
\text { Paralizadas }=\int(N P-R C) d t
$$

La ecuación 3 define la variable de nivel materiales que representa la acumulación, en toneladas, de los flujos de entrada netos de materiales de construcción; es decir, demanda de materiales del sector de construcción (D) menos materiales reciclados (R) y desechados como residuos de demolición (RE).

$$
\text { Materiales }=\int D-(R+R E) d t
$$

El nivel de materiales se relaciona con la construcción a través de la intensidad del consumo de materiales que es un factor que depende de la tecnología, en este caso definida como técnica constructiva.

$$
\text { Material }_{i}=\sum_{j} \text { Intensidad }_{i j}\left[\mathrm{~kg} / \mathrm{m}^{2}\right] * \text { porcentaje }_{j} * \text { área construida }{ }_{j}\left[\mathrm{~m}^{2}\right]
$$

Donde j: Técnica constructiva

$$
\text { i: Materiales }
$$

\subsection{Proyección de variables exógenas}

Para proyectar las variables exógenas PIB, PIB construcción y construcción y edificaciones se ajustaron modelos auto-regresivos univariados de medias móviles Arima y Sarima.

Los modelos Arima se usan para pronosticar series estacionarias (o que se pueden convertir en estacionarias diferenciándolas). Un modelo de pronóstico Arima (Modelo Auto-regresivo Integrado de Media Móvil) es una ecuación lineal en la que los predictores son retardos de la variable dependiente (términos $A R$ ) y / o retardos del error 
de pronóstico (términos $M A$ ). Un modelo no estacional Arima $(p, d, q)$ tiene $p$ retardos de la variable dependiente, necesita $d$ diferenciaciones para ser estacionario, y tiene $q$ retardos del error de pronóstico.

El modelo estacional Sarima $(p, d, q)(P, D, Q) s$ representa tanto las variaciones estacionales de la serie como el componente regular. El componente regular es el primer término, modelo $(p, d, q)$. El segundo término tiene en cuenta los movimientos estacionales, donde $P$ es el número de términos auto-regresivos estacionales SAR, $D$ es el número de diferencias estacionales y $Q$ es el promedio móvil estacional [25]; s representa la periodicidad de la serie de tiempo; en este caso $s=4$ porque las series son trimestrales.

En este estudio se emplean modelos Arima y Sarima porque los datos son trimestrales y tienen variaciones estacionales de cuatro períodos (ver tablas 2 y 3 ). Para proyectar la variable exógena PIB, la cual influye en el número de obras iniciadas [6], se ajustó un modelo Sarima $(0,1,0)(4,0,5) 4$ con constante (ecuación 5). De igual manera, se ajustó un modelo Sarima $(0,0,0)(4,0,5) 4$ con constante para la Variación de Construcciones y edificaciones (ecuación 6). Para el PIB-construcción, se tomó un modelo Arima $(2,1,4)$ (ecuación 7 ). Las proyecciones se hicieron con datos trimestrales entre 2000-I y 2014-III por CAMACOL [16]; en la tabla 2 se muestran las pruebas realizadas a los modelos de las ecuaciones 4 a 6 . Las ecuaciones de los modelos ajustados son las siguientes:

$$
\begin{gathered}
P I B_{t}=c+\sum_{i=1}^{4} \alpha_{i} P I B_{t-i s}+\sum_{j=1}^{5} \beta_{j} P I B_{t-j s}+V_{t} \\
C E_{t}=c+\sum_{i=1}^{4} \alpha_{i} C E_{t-i s}+\sum_{j=1}^{5} \beta_{j} C E_{t-j s} \\
P I B C_{t}=\sum_{i=1}^{2} \alpha_{i} P I B C_{t-i}+\sum_{j=1}^{4} \beta_{j} P I B C_{t-j}+V_{t}
\end{gathered}
$$

\begin{tabular}{|c|c|c|c|}
\hline \multicolumn{4}{|c|}{ Prueba de Aleatoriedad de residuos } \\
\hline Variable de datos & Var PIB.Col_2 & PIB_const.Col_2 & varCe.Col_2 \\
\hline Modelo: & $\begin{array}{c}\text { Sarima }(0,1,0) \\
\quad(4,0,1) 4\end{array}$ & $\begin{array}{l}\text { Arima }(2,1,4) \\
\text { con constante }\end{array}$ & $\begin{array}{c}\text { Sarima }(0,0,0) \\
\mathrm{x}(4,0,5) 4 \text { con } \\
\text { constante }\end{array}$ \\
\hline
\end{tabular}

Tabla 2: Pruebas de los modelos Arima y Sarima (ecuaciones 5 a 7) 


\begin{tabular}{|c|c|c|c|}
\hline \multicolumn{4}{|c|}{ Prueba de Aleatoriedad de residuos } \\
\hline Variable de datos & Var PIB.Col_2 & PIB_const.Col_2 & varCe.Col_2 \\
\hline \multicolumn{4}{|l|}{ (1) Corridas arriba o abajo de la mediana } \\
\hline Mediana & 0,000700083 & 0,000279176 & 0,0244476 \\
\hline Número de corridas arriba/ abajo de la mediana & 29 & 30 & 28 \\
\hline Número esperado de corridas & 27,0 & 27,0 & 28,0 \\
\hline Estadístico z para muestras grandes & 0,420165 & 0,700275 & 0,137385 \\
\hline Valor-P & $0,67436^{*}$ & 0,483753 & 1,0 \\
\hline \multicolumn{4}{|l|}{ (2) Corridas arriba y abajo } \\
\hline Número de corridas & 40 & 34 & 36 \\
\hline Número esperado de corridas & 35,0 & 35,0 & 356.667 \\
\hline Estadístico z para muestras grandes & 149.174 & 0,165748 & $-0,0547176$ \\
\hline Valor-P & $0,135768^{*}$ & 0,86835 & 1,0 \\
\hline \multicolumn{4}{|l|}{ (3) Prueba Box-Pierce } \\
\hline Prueba basada en número de autocorrelaciones & 17 & 17 & 18 \\
\hline Estadístico de prueba para muestras grandes & 12,9282 & 13,3587 & 11,0658 \\
\hline Valor-P & $0,374284^{*}$ & $0,270527^{*}$ & $0,271222^{*}$ \\
\hline
\end{tabular}

*No se puede rechazar la hipótesis nula de que los residuos son aleatorios, es decir hay ruido blanco Fuente: elaboración propia

Tabla 3: Pronósticos Construcción y edificaciones, PIB construcción, PIB (ecuaciones 5 a 7)

\begin{tabular}{|c|c|c|c|c|c|c|}
\hline Variable & \multicolumn{2}{|c|}{ varCe.Col_2 } & \multicolumn{2}{|c|}{ PIB_const.Col_2 } & \multicolumn{2}{|c|}{ Var PIB.Col_2 } \\
\hline Observaciones & \multicolumn{2}{|c|}{54} & \multicolumn{2}{|c|}{54} & \multicolumn{2}{|c|}{54} \\
\hline Estacionalidad & \multicolumn{2}{|c|}{4} & \multicolumn{2}{|r|}{4} & \multicolumn{2}{|c|}{4} \\
\hline Ajuste estacional: & \multicolumn{2}{|c|}{ Multiplicativo } & & & \multicolumn{2}{|c|}{ Multiplicativo } \\
\hline Modelo & \multicolumn{2}{|c|}{$\begin{array}{c}\text { Sarima }(0,0,0) \times(4,0,5) 4 \\
\text { con constante }\end{array}$} & \multicolumn{2}{|c|}{$\operatorname{Arima}(2,1,4)$ con constante } & \multicolumn{2}{|c|}{$\operatorname{Sarima}(0,1,0) \times(4,0,1) 4$} \\
\hline Pronósticos & \multicolumn{2}{|c|}{15} & \multicolumn{2}{|c|}{15} & \multicolumn{2}{|c|}{15} \\
\hline Periodos-validación & \multicolumn{2}{|c|}{8} & \multicolumn{2}{|r|}{8} & \multicolumn{2}{|c|}{8} \\
\hline Estadístico/Periodo & Estimación & Validación & Estimación & Validación & Estimación & Validación \\
\hline RMSE & 0,0841 & 0,1299 & 0,0026 & 0,0057 & 0,00804 & 0,0127 \\
\hline MAE & 0,0596 & 0,1053 & 0,0018 & 0,0046 & 0,0059 & 0,0086 \\
\hline
\end{tabular}




\begin{tabular}{|c|c|c|c|c|c|c|}
\hline Variable & \multicolumn{2}{|c|}{ varCe.Col_2 } & \multicolumn{2}{|c|}{ PIB_const.Col_2 } & \multicolumn{2}{|c|}{ Var PIB.Col_2 } \\
\hline MAPE & $\leq 0$ & $\leq 0$ & 3,18851 & 6,7593 & 20,0501 & 30,4092 \\
\hline ME & 0,0102125 & 0,0309989 & $\leq 0$ & 0,00069 & 0,00077 & $-0,0028$ \\
\hline MPE & $\leq 0$ & $\leq 0$ & 0,046571 & 0,3533 & $-8,2896$ & $-16,4186$ \\
\hline Pronóstico histórico & \multicolumn{2}{|c|}{ Sí } & \multicolumn{2}{|c|}{ Sí } & \multicolumn{2}{|c|}{ Sí } \\
\hline Varianza-ruido blanco & \multicolumn{2}{|c|}{0,0079} & \multicolumn{2}{|c|}{0,00000722} & \multicolumn{2}{|c|}{0,00007200} \\
\hline Grados de libertad & \multicolumn{2}{|c|}{44} & \multicolumn{2}{|c|}{46} & \multicolumn{2}{|c|}{48} \\
\hline SD-ruido blanco & \multicolumn{2}{|c|}{0,0888883} & \multicolumn{2}{|c|}{0,00268721} & \multicolumn{2}{|c|}{0,00848875} \\
\hline Iteraciones & \multicolumn{2}{|c|}{36} & \multicolumn{2}{|c|}{8} & \multicolumn{2}{|c|}{4} \\
\hline Valores P & \multicolumn{2}{|c|}{$<0.5$} & \multicolumn{2}{|c|}{$<0.5$} & \multicolumn{2}{|c|}{$<0.5$} \\
\hline Criterio de selección & \multicolumn{2}{|c|}{ Akaike } & \multicolumn{2}{|c|}{ Akaike } & \multicolumn{2}{|c|}{ Akaike } \\
\hline
\end{tabular}

Fuente: elaboración propia

\section{SIMULACIÓN DE LA CONSTRUCCIÓN DE EDIFICACIONES Y DEMANDA DE MATERIALES}

La actividad edificadora se simula para un horizonte de tiempo de cuatro años, entre 2014-I y 2018-I, con un paso de tiempo trimestral, acorde con el reporte de los Censos de Edificaciones DANE [23]. Las variables exógenas de crecimiento económico se proyectan usando los modelos descritos en la sección 2.2. Las proyecciones se muestran en las figuras 4,5 y 6 .

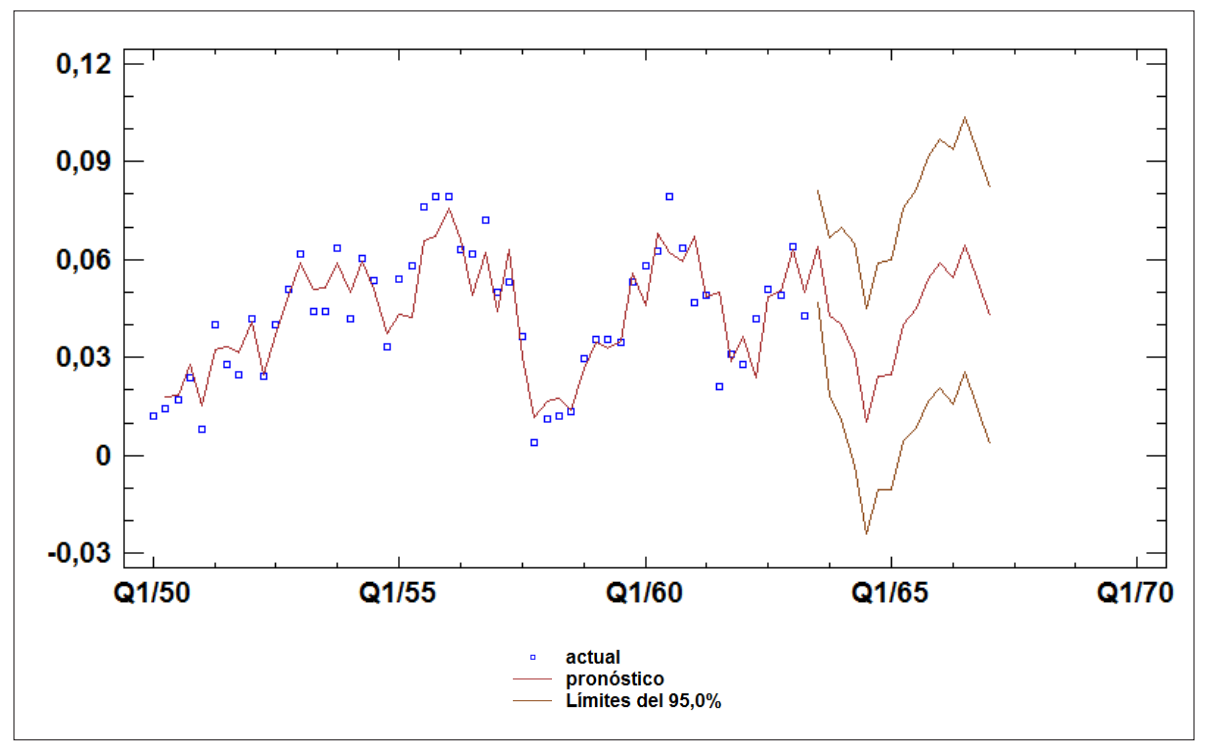

Figura 4: Pronóstico variación PIB

Fuente: elaboración propia a partir de modelo Sarima $(0,1,0)(4,0,5) 4$ (Ecuación 5) y datos de DANE [15] 


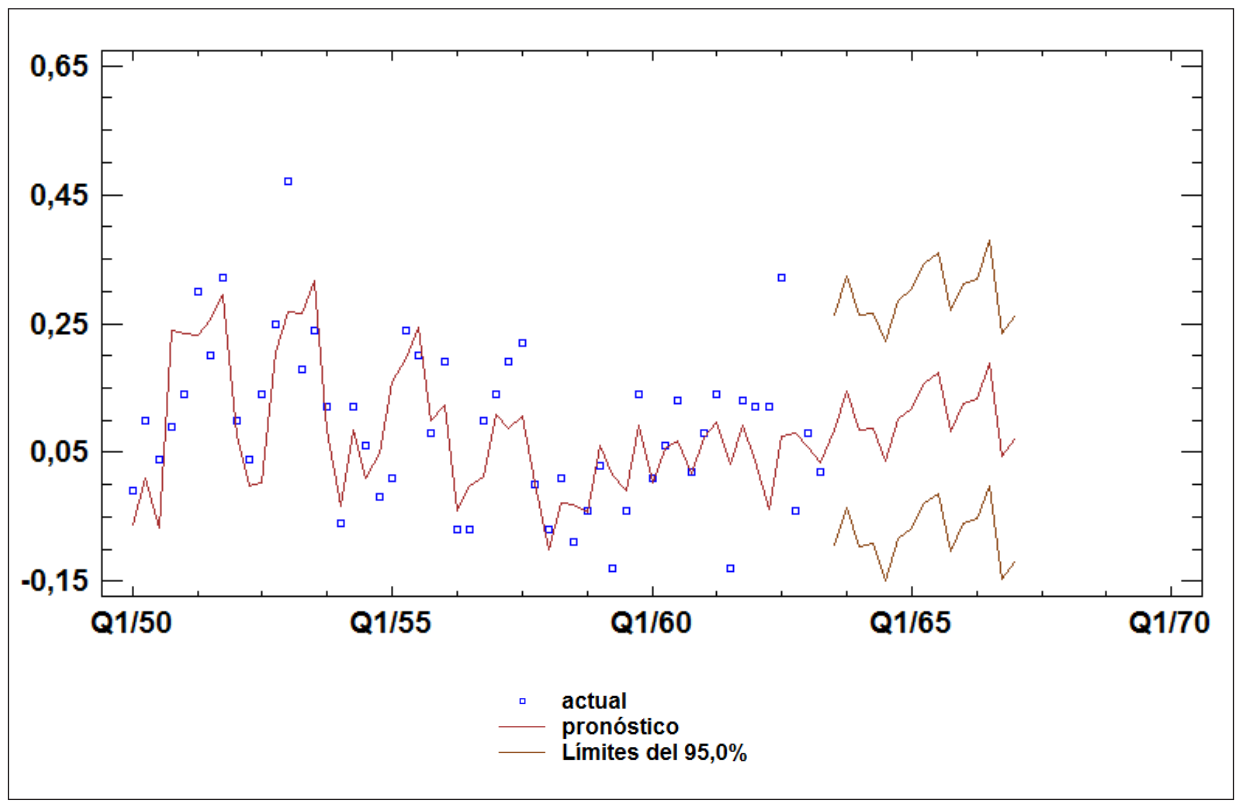

Figura 5: Pronóstico variación construcciones y edificaciones.

fuente: elaboración propia a partir de modelo Sarima $(0,0,0)(4,0,5) 4$ (Ecuación 6) y datos de DANE [15]

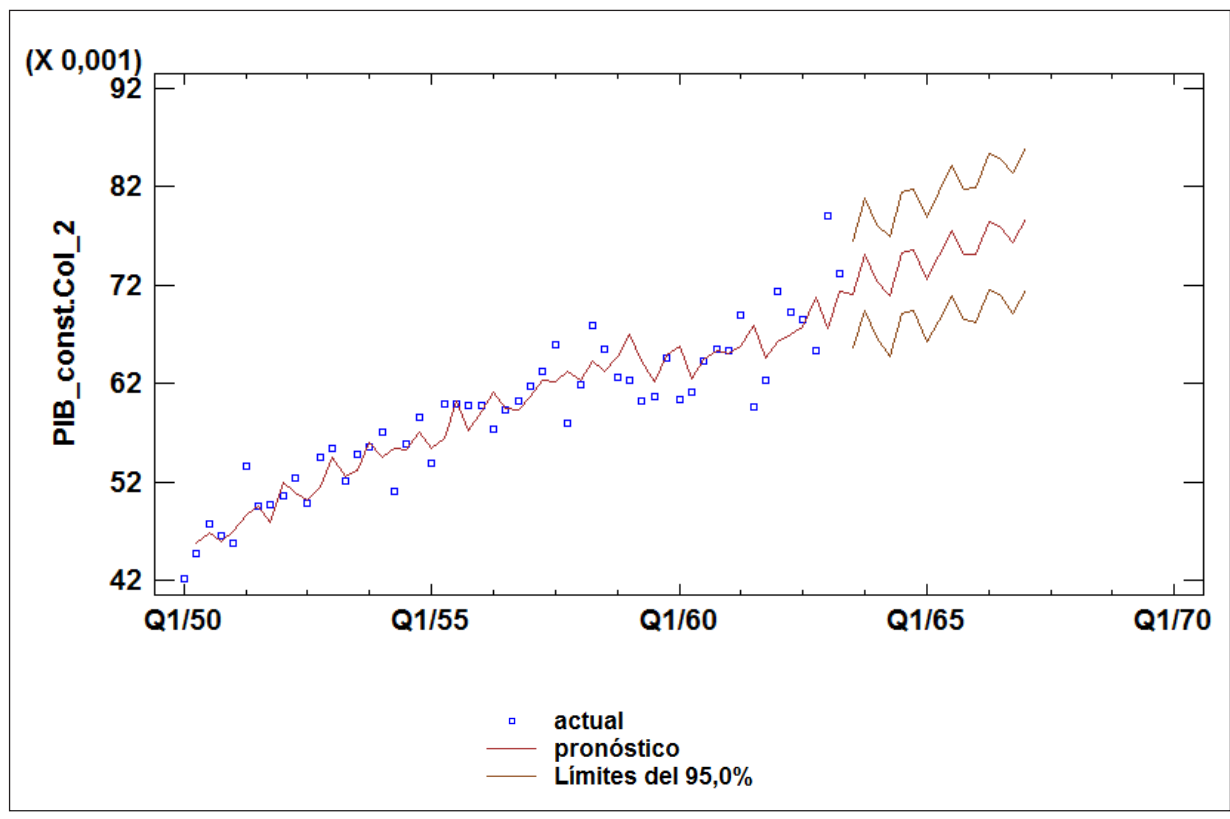

Figura 6: Pronóstico variación PIB-construcción.

Fuente: elaboración propia a partir de modelo Arima $(2,1,4)$ (ecuación 7) y datos de DANE [15]. 
Además, en la tabla 4 se tienen las condiciones iniciales para el modelo de dinámica de sistemas presentado en la sección 2.1

Tabla 4: Valores iniciales del Modelo en Dinámica de sistemas

\begin{tabular}{|c|c|c|c|}
\hline Variable & Valor & Unidad & Fuente \\
\hline En construcción & 20.439 .315 & $\mathrm{~m}^{2}$ & [2] \\
\hline Paralizadas & 3.795 .786 & $\mathrm{~m}^{2}$ & {$[2]$} \\
\hline Obras nuevas iniciales & 4.581 .655 & $\mathrm{~m}^{2}$ & [2] \\
\hline Materiales & $51.653,1$ & Millones de toneladas & Cálculos propios \\
\hline Parámetros & Valor & Unidad & Fuente \\
\hline $\begin{array}{l}\text { Participación Mconfinada en } \\
\text { obras iniciadas }\end{array}$ & 19,22 & $\%$ & [2], [27] Cálculos propios \\
\hline $\begin{array}{l}\text { Participación Mestructural en } \\
\text { obras iniciadas }\end{array}$ & 2,70 & $\%$ & [2], [27] Cálculos propios \\
\hline $\begin{array}{l}\text { Participación Mconfinada en } \\
\text { obras iniciadas }\end{array}$ & 76,84 & $\%$ & [2], [27] Cálculos propios \\
\hline Tasa reciclaje & 5 & $\%$ & {$[28]$} \\
\hline Tasa residuos & 5 & $\%$ & {$[28]$} \\
\hline Tiempo de demolición & 47 & trimestres & Calibrado \\
\hline Tasa obsolescencia & 92,47 & trimestres & Calibrado \\
\hline Tasa obras culminadas & 17,04 & $\% /$ trimestre & {$[2]$} \\
\hline Tasa obras paralizadas & 4,96 & $\% /$ trimestre & [2] \\
\hline Tasa obras reinicio & 12,13 & $\% /$ trimestre & [2] \\
\hline
\end{tabular}

Fuente: elaboración propia

Como se observa en la figura 7, en 2018-I se alcanzarían 28 millones de $\mathrm{m}^{2}$ en construcción con unos 9 millones de $\mathrm{m}^{2}$ en obras paralizadas. Esta tendencia se explica porque el flujo de obras iniciadas, que fluctúa entre 4.6 y 5.3 millones de metros cuadrados $\left(\mathrm{m}^{2}\right)$ trimestralmente, es mayor que el flujo de obras culminadas.

Las obras paralizadas tienden a crecer porque la tasa de reanudación de construcciones paralizadas es menor que la tasa de suspensión de obras.

Con el fin de estimar las cantidades de materiales de construcción demandados en el periodo de análisis, se usan los valores de la tabla 5 donde se detalla el consumo de cada material $\left(\mathrm{en} \mathrm{kg} / \mathrm{m}^{2}\right.$ ) para los principales sistemas constructivos usados en Colombia. 


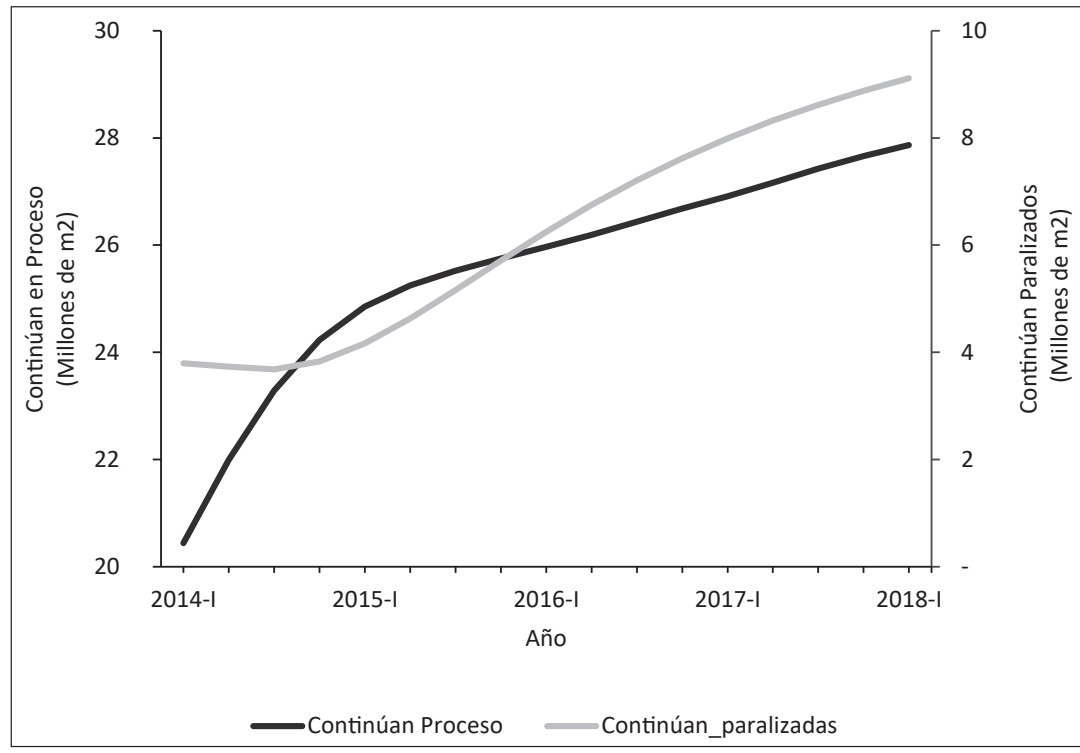

Figura 7: Simulación de obras en proceso de construcción y paralizadas Fuente: elaborado a partir de resultados de simulación (ecuaciones 1 a 3).

Tabla 5: Materiales de construcción por sistema constructivo

\begin{tabular}{|l|c|c|c|}
\hline \multirow{4}{*}{ Materiales } & $\begin{array}{c}\text { Sistemas } \\
\text { Industrializados }\end{array}$ & $\begin{array}{c}\text { Mampostería } \\
\text { Estructural }\end{array}$ & $\begin{array}{c}\text { Mampostería } \\
\text { Confinada }\end{array}$ \\
\cline { 2 - 4 } Agregados triturados & $\mathrm{kg} / \mathrm{m}^{2}$ & $\mathrm{~kg} / \mathrm{m}^{2}$ & $\mathrm{~kg} / \mathrm{m}^{2}$ \\
Arena de río & 542,19 & 404,05 & 624,99 \\
Cemento gris & 445,21 & 349,09 & 733,63 \\
roca muerta-tierra excavación & 156,74 & 138,78 & 306,12 \\
Cerámica cocida & 46,60 & 152,24 & 372,52 \\
Otros & 39,98 & 301,28 & 358,08 \\
\hline Total & 38,31 & 33,03 & 11,96 \\
\hline
\end{tabular}

Fuente: Salazar [27]

La mampostería confinada ha sido el sistema constructivo más implementado en Colombia con una participación en el 2014-III de 54\%, seguido por los sistemas industrializados y la mampostería estructural con $35,9 \%$ y $9,5 \%$, respectivamente. Sin embargo, los sistemas industrializados han ido ganando más peso al pasar 9,3\% en 2004 a 35,9\% en 2014, al tiempo que ha disminuido la aplicación de la mampostería estructural [16]. 
En la tabla 6 se comparan las proyecciones de demanda de materiales de construcción para Colombia entre 2014 y 2017 sin demoliciones, y asumiendo tasas de demolición y de reciclaje del 5\% [26]. Estas proyecciones se obtienen a partir de la simulación del modelo y de los consumos de materiales de la tabla 5.

Tabla 6: Proyección de demanda anual de materiales de construcción para viviendas y edificaciones en Colombia.

\begin{tabular}{|c|c|c|c|c|c|c|c|c|c|c|c|c|}
\hline & \multirow{2}{*}{\multicolumn{2}{|c|}{$\begin{array}{c}\begin{array}{c}\text { Agregados } \\
\text { triturados }\end{array} \\
\text { Reciclaje }\end{array}$}} & \multirow{2}{*}{\multicolumn{2}{|c|}{$\begin{array}{c}\text { Arena de rios } \\
\text { Reciclaje }\end{array}$}} & \multirow{2}{*}{\multicolumn{2}{|c|}{$\begin{array}{c}\text { Cemento gris } \\
\text { Reciclaje }\end{array}$}} & \multirow{2}{*}{\multicolumn{2}{|c|}{$\begin{array}{c}\text { Roca muerta } \\
\text { Reciclaje }\end{array}$}} & \multirow{2}{*}{\multicolumn{2}{|c|}{$\begin{array}{c}\text { Cerámica } \\
\text { cocida } \\
\text { Reciclaje }\end{array}$}} & \multirow{2}{*}{\multicolumn{2}{|c|}{$\begin{array}{c}\text { Otros } \\
\text { Reciclaje }\end{array}$}} \\
\hline & & & & & & & & & & & & \\
\hline & No & Sí & No & Sí & No & Sí & No & Sí & No & Sí & No & Sí \\
\hline 2014 & 12,32 & 11,44 & 13,63 & 12,66 & 5,57 & 5,17 & 6,19 & 5,75 & 6,02 & 5,59 & 0,36 & 0,34 \\
\hline 2015 & 12,17 & 11,24 & 13,47 & 12,44 & 5,50 & 5,08 & 6,12 & 5,65 & 5,95 & 5,49 & 0,36 & 0,33 \\
\hline 2016 & 12,41 & 11,13 & 13,72 & 12,31 & 5,61 & 5,03 & 6,24 & 5,59 & 6,06 & 5,44 & 0,36 & 0,33 \\
\hline 2017 & 12,54 & 10,97 & 13,88 & 12,14 & 5,67 & 4,96 & 6,30 & 5,51 & 6,13 & 5,36 & 0,37 & 0,32 \\
\hline Total & 49,43 & 44,78 & 54,69 & 49,54 & 22,34 & 20,24 & 24,85 & 22,51 & 24,16 & 21,89 & 1,45 & 1,31 \\
\hline
\end{tabular}

Fuente: cálculos propios. Cifras en millones de toneladas

Para el período de simulación, la demanda de materiales de construcción para la actividad edificadora sería de 176,9 millones de toneladas sin reutilizar materiales demolidos y de 160,3 millones de toneladas reutilizando materiales. Este 10,4\% de diferencia (16,6 millones de toneladas), que se muestra en la figura 8 , ilustra el potencial de la oferta secundaria de materiales de construcción.

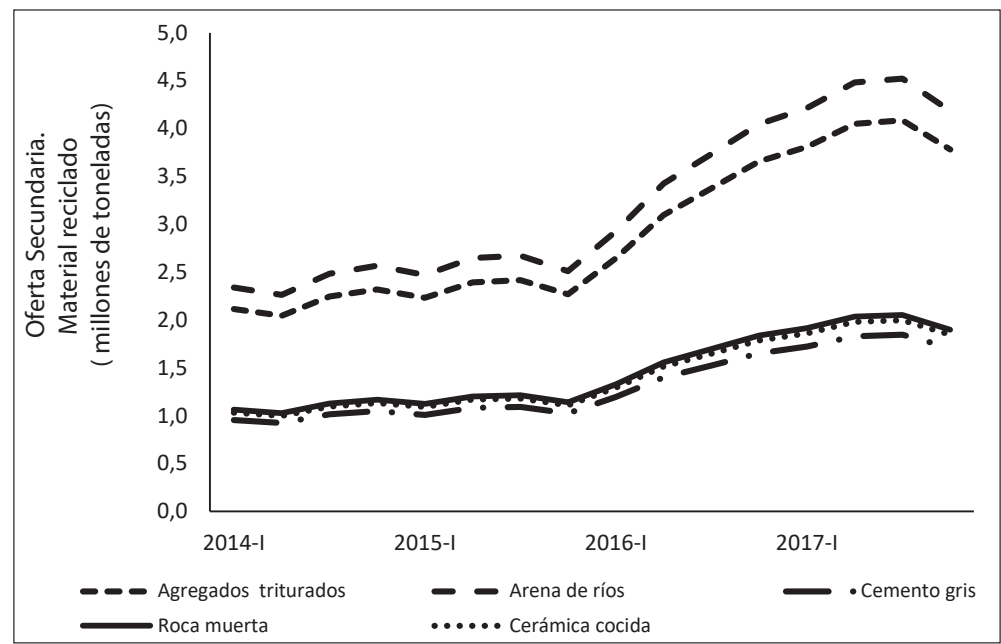

Figura 8: Estimación de la oferta secundaria de materiales de construcción reciclados a partir de resultados de simulación (ecuaciones 1 a 4). 
Para estimar la cantidad de materias primas requeridas para fabricar el cemento y concreto demandados por la construcción de viviendas, se usan los flujos de materiales de la figura 1. Como se observa en la tabla 7, entre 2014 y 2017 la fabricación de cemento requeriría aproximadamente 52,54 millones de toneladas de materiales. Estas proyecciones se hacen sin tener en cuenta la oferta secundaria. Sin contar con demoliciones, y asumiendo que la participación de los sistemas constructivos se mantiene como en la tabla 4, entre 2014 y 2017 se necesitarían 9,31 y 14,95 millones de toneladas de arena y grava como insumo para la preparación de concreto.

Tabla 7: Demanda de minerales de construcción estimada a partir de la demanda de cemento gris para viviendas y edificaciones sin demoliciones.

\begin{tabular}{|l|c|c|c|c|c|c|}
\hline \multirow{2}{*}{ Año } & \multicolumn{7}{|c|}{$\begin{array}{c}\text { Minerales para construcción } \\
\text { (millones de toneladas, sin demoliciones) }\end{array}$} \\
\cline { 2 - 7 } & Caliza & Yeso & Arcilla & Minerales de hierro & Arena & Grava \\
\hline 2014 & 3,83 & 3,52 & 3,32 & 2,42 & 2,32 & 3,73 \\
\hline 2015 & 3,79 & 3,47 & 3,28 & 2,39 & 2,29 & 3,68 \\
\hline 2016 & 3,86 & 3,54 & 3,35 & 2,44 & 2,34 & 3,75 \\
\hline 2017 & 3,90 & 3,58 & 3,38 & 2,46 & 2,36 & 3,79 \\
\hline Total sin reciclaje & 15,38 & 14,11 & 13,33 & 9,71 & 9,31 & 14,95 \\
\hline Total con reciclaje & 13,94 & 12,78 & 12,07 & 8,80 & 8,44 & 13,55 \\
\hline
\end{tabular}

**** Se calculó multiplicando la producción en $\mathrm{m}^{3}$ por $2.550 \mathrm{~kg} / \mathrm{m}$

Al comparar el área en construcción simulada con la reportada por el DANE para el trimestre 4 de 2014 y trimestre 4 de 2015, se encuentra que el área en construcción simulada es un 3\% mayor que la reportada por el DANE para el trimestre 4 de 2014 y $10 \%$ mayor que la reportada para el mismo trimestre del 2015. Las diferencias se atribuyen, en parte, a errores en el pronóstico de las variables exógenas.

\section{CONCLUSIONES}

El análisis de flujos de materiales de construcción indica que la mayor parte de la producción de cemento y concreto se destina a la construcción de edificaciones. En este artículo mostramos cómo es posible estimar el flujo de materiales para la construcción de edificaciones a partir de la dinámica de la construcción.

Las estadísticas oficiales registran los cambios en la producción de calizas y cemento, pero no hay un registro de arenas, gravas y otros materiales usados en grandes 
volúmenes. El análisis de flujos de materiales presentado en este artículo permite estimar la cantidad de arcillas, arenas y gravas, entre otros materiales usados en la producción de cemento y concreto para el sector de la construcción.

Al igual que en otros estudios de proyección de demanda de materiales, este trabajo incluye variables económicas y modelos econométricos para explicar la relación entre el crecimiento económico y la actividad constructora. Asimismo, se tiene en cuenta el consumo de materiales de las principales técnicas constructivas con el fin de calcular la demanda de materiales de construcción a partir de la actividad constructora.

El trabajo presentado contribuye al estudio de la demanda de materiales de construcción en Colombia porque permite estimar la demanda de minerales y materiales de construcción teniendo en cuenta las tecnologías para su transformación y uso, y porque incluye otras variables que definen el comportamiento dinámico de la construcción, como son los retardos de construcción y la oferta secundaria de materiales.

La principal dificultad en la aplicación de la metodología presentada está en la disponibilidad de fuentes de información. En este caso, el análisis se limitó a los materiales requeridos para apoyar la construcción de edificaciones reportada por el DANE. No obstante, la metodología es lo suficientemente flexible como para aplicarse también a la construcción de infraestructura.

Entre los trabajos futuros posibles está el desarrollo de un modelo de largo plazo de la dinámica de la construcción que permita evaluar el efecto de, por ejemplo, cambios tecnológicos en la construcción y de cambios sociales y demográficos sobre la actividad constructora. Otras extensiones incluyen la evaluación de los consumos de energía y agua en los procesos de transformación de materias primas y construcción, así como un análisis más detallado de los factores que influyen en el reciclaje de materiales de demolición.

\section{REFERENCIAS}

[1] ACRIP-FEDESARROLLO, "Informe Mensual del Mercado Laboral. Composición sectorial del empleo en Colombia”, Formas Finales Ltda., Bogotá, 2013.

[2] DANE, “Construcción”, 2015a. [En línea]. Available: http://goo.gl/Eq9hNG. [Último acceso: 26 Julio 2014].

[3] DANE, "Encuesta Anual Manufacturera", DANE, 2015b. [En línea]. Available: http://goo.gl/ w3qilb. [Último acceso: marzo 2015].

[4] LR, “Auge de los materiales para la construcción”, La República, Bogotá, 2014.

[5] Argos, "Proyectos de expansión de capacidad de cemento en el centro de Colombia", 18 septiembre 2012. [En línea]. Available: http://goo.gl/UL0vZ3.. [Último acceso: 15 julio 2015]. 
[6] UPME, "Evaluación de la situación actual y futura del mercado de los materiales de construcción y arcillas en las ciudades de Bogotá, Medellín, Bucaramanga, Barranquilla, Santa Marta y eje cafetero", Bogotá, 2014.

[7] R. Ayres y A. Kneese, "Production, Consumption, and Externalities", American Economic Review, vol. 59, n. 3 , pp. 282-96, 1969.

[8] Eurostat, "Economy-wide material flow accounts and derived indicators: A methodological guide", Office for Official Publications of the European Communities, Luxembourg, 2001.

[9] Eurostat, "Economy-wide material flow accounting: A compilation guide", Eurostat, Luxembourg, 2012.

[10] J. Sterman, Business Dynamics. Systems thinking and modeling for a complex world, Massachusetts: Irwin McGraw-Hill, 2000.

[11] P. Brunner y H. Rechberger, Practical Handbook of Material Flow Analysis, Boca Raton, FL: CRC / Lewis, 2004.

[12] OECD, "Measuring material flows and resource productivity", OECD, 2008.

[13] M. C. Vallejo, M. A. Pérez y J. Martínez-Alier, "Metabolic profile of the Colombian economy from 1970 to 2007”, Journal of industrial Ecology, vol. 15, n. . 2, pp. 245-267, 2011.

[14] M. Pidd, Computer Simulation in Management Science, Chichester : John Wiley and Sons, Ltd, 2004.

[15] DANE, “Departamento Administrativo Nacional de Estadística", 2014. [En línea]. Available: http://www.dane.gov.co/index.php/estadisticas-por-tema/construccion. [Último acceso: 24 octubre 2014].

[16] CAMACOL, “Construcción en Cifras", febreo 2015. [En línea]. Available: http://goo.gl/ NhJSpE. [Último acceso: abril 4 2015].

[17] SIMCO, "SIMCO”, 2014. [En línea]. Available: http://www.simco.gov.co/?TabId=121. [Último acceso: 24 octubre 2014].

[18] EPA, “National Waste Report 2007”, Environmental Protection AgencY, Wexford, 2007.

[19] WBCSD, “The Cement Sustainability Initiative: Cement Industry Energy and CO2 Performance "Getting the Numbers Right"", World Business Council for Sustainable Development, Washington, DC, 2009.

[20] EPA, "Study on Increasing the Usage of Recovered Mineral Components in Federally Funded Projects Involving Procurement of Cement or Concrete", Environmental Protection Agency, 2008.

[21] PCA, “America’s Cement Manfactures", 2014. [En línea]. Available: http://www.cement.org/ cement-concrete-basics/how-concrete-is-made. [Último acceso: 24 octubre 2014]. 
[22] DANE, Metodología Estadísticas de Cemento Gris, Bogotá: Imprenta Nacional de Colombia, 2011a.

[23] DANE, "Ficha Metodológica Censo de Edificaciones-CEED”, 2011b. [En línea]. Available: http://goo.gl/xxPIzO.

[24] M. Cárdenas, X. Cadena y J. F. Quintero, "Determinantes de la actividad constructora en Colombia", Fedesarrollo, Bogotá, 2004.

[25] W. Enders, Applied Econometric Times Series, New York: John Wiley \& Sons., 2008.

[26] J. O. Castaño, L. A. Lasso, M. R. Rodríguez, A. Gómez Cabrera y M. S. Ocampo, “Gestión de residuos de construcción y demolición (RCD) en Bogotá: perspectivas y limitantes”, Pontificia Universidad Javeriana, Bogotá, 2013.

[27] M.-S. Low, "Material Flow Analysis of Concrete in the United States”, MIT, Massachusetts, 2005.

[28] A. Salazar, "Determinación de las propiedades físicas y, estimación del consumo energético en la producción, de acero, concreto, vidrio, ladrillo y otros materiales, entre ellos los alternativos y otros de uso no tradicional", Ecoingeniería, Cali, 2013. 